Moroccan J. of Pure and Appl. Anal. (MJPAA)

Volume 5(1), 2019, Pages 37-45

ISSN: Online 2351-8227 - Print 2605-6364

DOI 10.2478/mjpaa-2019-0004

\title{
A Modified Fractional Differential Love Model
}

\author{
A. T. Talabi ${ }^{1, a}$, R. K. Odunaike ${ }^{1, b}$, O. S. Odetunde ${ }^{2, c}$ and K. A. Omoteso ${ }^{1, d}$
}

\begin{abstract}
Aвstract. A modified system of nonlinear fractional-order differential equations become used to classify humans of various personalities and different Impact Factors of Memory (IFM); with unique set of model parameters. The model was used to interpret and predict the functions of the union of various people with external circumstance(s) and adapted to neighborhood environment in which the statistics collections were achieved to analyze numerous measures affecting marriages, unique challenges in marriage and associated occasions were investigated through the use of questionnaire. Data had been analyzed and the outcomes have been carried out as parameters to validate the model. Adams Predictor-Corrector Method was used to test the chaoticity of the system and it was confirmed via numerical simulations.

Numerical simulation outcomes had been presented to reveal the effectiveness of the model and the accuracy of the statements established. The mathematical information implied by the model unveil an underlying mechanism which can give an explanation for couple disruption in relationships that were initially deliberated to remain all the time. Despite the terrible aspects of relationships, some human beings were still satisfied in their intimate members of the family. The study was addressed on a field survey (use of questionnaires) and with the aid of interrogating the members one on one. A feasible path for future work is the choice to attain balance through modelling and to validate the results with the aid of numerical simulations.

Key words and phrases. Science; Impact factor memory; marriages; fractional differential equations; Adams predictor corrector approach; relationship.
\end{abstract}

Received May 08, 2019 - Accepted July 29, 2019.

(C)The Author(s) 2017. This article is published with open access by Sidi Mohamed Ben Abdallah University.

${ }^{1}$ Department of Physics, Olabisi Onabanjo University, Ago-Iwoye, Nigeria.

2 Department of Mathematics,Olabisi Onabanjo University, Ago-Iwoye, Nigeria

a e-mail: talabi.adetoro@oouagoiwoye.edu.ng

${ }^{b}$ e-mail:kolaodunaike@oouagoiwoye.edu.ng

c e-mail: tunde.odetunde@oouagoiwoye.edu.ng

d e-mail: omotesokehinde@physics.org . 


\section{INTRODUCTION}

The mathematics to the study of marriage turned into presaged with the aid of Von Bertalanfy [14] and the study has begun to hold both the artistic imaginations and interest of diverse scholars inside the fields of mathematics, physics, sociology, biology, neuroscience, psychology and anthropology [2]. Application of mathematical power to the dynamics of marriage has laid basis for a systematic idea of marital relations [3]. Marriage may be known as a relationship ruled by way of comments, or circular causality, in which every person continually responds to the alternative in predictable ways that maintain styles of interacting in the system however the presence of chaos in marriage is unwanted [15]. Marriage is tough to quantify, therefore make experiment on this area difficult to layout.

However, earthly observations are the most effective sources of description of such relationship expressed within individuals, groups or set of people. It may be inferred that mathematical models can also play a first-rate position in reading dynamics of marriage via the usage of a set of nonlinear and linear differential equations, manipulating techniques in stabilizing chaos in marriage, promoting a peaceful co-existence and displaying the behavioural time collection and section plots [12]. Modelling dynamical system or phenomena in life sciences, economics, engineering, specifically in marriage dynamics the use of classical integer order differential equations has attracted researchers several years in the past. One of the earliest efforts at modelling marital relationships was by a group of researchers and mathematicians who teamed who to create a mathematical model that intended to predict future of marriages using a set of classical integer first order differential equations, but there's a restrict to integer order models because love is a state that is continually stimulated with the aid of historical statistics [7].

But due to the effectiveness of fractional order integrals and derivatives cannot be over-emphasized hence, numerous model in sciences and engineering had been formulated and analyzed efficiently [10]. Fractional order differential operator is non local. This makes fractional differential equation to be veritable tools for modelling marital relations. Gottman et.al [4] evolved a technique that makes use of both the data and the mathematics of differential equations to create a qualitative mathematical representation of change (section plots). Gu et.al [6] stated that applications of fractional order differential are recent research recognition of interest which can be used to describe lifestyles sciences and psychological tactics and they considered dynamical model with fractional-order and control problems of the fractional-order love system using feedback method for ODE systems. Okyere et al. [10] in their research work formulated an epidemiological model by use of fractional order derivatives which has an advantage over the classical integer order version because of its memory impact assets. Song et.al [13] carried out explorative studies on chaos control and synchronization of one's emotion. They used Adams predictor corrector scheme because of the specificity of the error estimation bound, performance and reliability; with this technique they were capable of discovering the existence of chaos within the fractional-order happiness model.

The fractional derivatives concept first appeared in a letter written to L'Hospital in 1695 from Leibniz. The theory of the study was first constrained to the field of pure theoretical mathematics three centuries ago which is beneficial to both the theoretical physicists and mathematicians only [8]. Also, it had been successfully used in modeling many physical phenomena these days together with nonlinear oscillation of earthquake, acoustics, electromagnetism, electrochemistry, diffusion system, fluid float, polymer physics, mathematical biology and numerous regions of technological know-how [1] , [9]. The study is a micro experiment whereby small sample size was determined according to specified model. The model used in this work is similar to that used by [11], it is a nonlinear dynamical system with two state variables. Demographic information in respect to the relationship length of participants recorded was used as parameter in the model but participants were inconsistent in the amount of demographic information provided. Not all questionnaire provided the necessary information for all the variables but the few that responded were picked for the analysis. 


\subsection{Couple dynamic equations: a modeling approach.}

The recent modeling approach has received some attention in scientific community. Gragnan et.al [5] assumed love to be composed of three terms and can be formulated by the equation.

$$
\dot{x}_{i}=O_{i}+R_{i}+I_{i}
$$

The Eqn. (1.1) includes lowest range of state variables. Three basic processes, namely oblivion $O_{i}$, return $R_{i}$ and $I_{i}$ instinct are assumed to be responsible of love dynamics. More precisely, the instantaneous rate of change $\dot{x}_{i}$. Eqn. (1.1) can be re-written in the form given in Eqn. (1.2)

$$
\begin{aligned}
& \dot{x}_{1}(t)=\beta_{1} x_{2}(t)+\gamma_{1} A_{2}-\alpha_{1} x_{1}(t) \\
& \dot{x}_{2}(t)=\beta_{2} x_{1}(t)+\gamma_{2} A_{1}-\alpha_{2} x_{2}(t)
\end{aligned}
$$

Eqn. (1.2) has two state variables $x_{1}$ (wife) and $x_{2}$ (husband) which are measures of the love of individuals, $\alpha_{i}, \beta_{i}, \gamma_{i}$ and $A_{i}$ are constant and positive parameters. The equations can be related to the coupled harmonic oscillator. Ideal couple can be related to as a physical system (physical system is a portion of the universe that has been chosen for studying or analysis) that when displaced from its equilibrium position, experiences a restoring force proportional to the displacement but when the elastic limit is exceeded therefore the system loses its elasticity and this can be referred to as chaotic couple. When there is chaos in the marriage, it tends to collapse the marriage but the bonding between the couple (children, achievements, e.t.c) can bring them back together. Let us recall that harmonic oscillator occurs widely in nature. It is a system that when displaced from its equilibrium position, experiences a restoring force $F$ which is virtually the source of sinusoidal vibrations and waves. This is where Newton's second law can be introduced.

$$
F=-k x
$$

Various forces may act on couple togetherness, which can be related to harmonic oscillator. These forces tend to reduce or increase the presence of chaos in family. Such forces can be romantic life styles, advice from friends, appeal and love of individual. Cheriff et.al [2], proposed a more general model on couples dynamics to show the behavioural features of romantic relationship, where only showed the phase plot of ideal relationship were presented $\epsilon=0$ as follows

$$
\begin{aligned}
& \frac{d X_{1}}{d t}=-\alpha_{1} X_{1}(t)+\beta_{1} X_{2}\left(1-\epsilon X_{2}^{2}\right)+A_{1} \\
& \frac{d X_{2}}{d t}=-\alpha_{2} X_{2}(t)+\beta_{2} X_{1}\left(1-\epsilon X_{1}^{2}\right)+A_{2}
\end{aligned}
$$

The model of consideration in this work is an improvement of the existing model in (4). The model was transited to fractional nonlinear differential equation that uses the result of the data analysis as its parameters. There are two major cases about marriage ;

Case I: It is either a marriage is in order or

Case II: A marriage is in disorder.

This work considered case II

1.2. Modified fractional model. The modified love model involves a periodic forcing term as shown

$$
\begin{aligned}
& \frac{d^{q} x}{d t^{q}}=-a x+b x\left(1-e y^{2}\right)+\sin f(t) \\
& \frac{d^{q} y}{d t^{q}}=-c y+d x\left(1-e x^{2}\right)+\sin f(t)
\end{aligned}
$$

For convenience of interpretation, some practical physical meanings were imposed on the parameters $a, b, c, d, e$ and $q$ of the above model (Eqn. 1.5). The variables are measure of emotions of individual $x$ and $y$ where 
$a, b, c, d, e, \sin f(t)$ are parameters and they are referred to as effect of: maltreatment, effect of loneliness, wrong advice, picking partners phone calls, influence and a time dependent forcing function representing ones external circumstances.

The parameters used in this study is to categorize people with different personality in marriage, the order $\mathrm{q}$ represents the Impact Factor of Memory (IFM) of the couple, that is, as the value of $q$ increases from 0 to 1, the IFM of an individual increases correspondingly.

\subsection{Simulation Results.}

\subsubsection{Result 1.}

In this paper numerical simulations were conducted on the modified fractional model using Adams predictor corrector approach (APCA). Different parameters of the couples were applied and the order varied. Also, different periodic function was used for result 1 and result two respectively because of different impact factor of memory, while the initial value is $(0.1,0.1)$.

Case 1: Time series and phase portraits of love system in Eqn. (1.5) with a periodic forcing for those that agreed with the questions being asked. For the couples that agreed with the questions asked at order 0.25 and 0.99, the trajectory of system in Eqn. (1.5) takes on periodicity as Fig. (2a) shows. If increases to 0.99, the system behave chaotically as shown in Fig. (1a) whereby the wife loves the husband, whereas the husband has hatred for the wife. Fig. (1b) shows the couple does not have feelings for each other as the year runs by and Fig. (2b) indicates the oscillations in the couples marriage.

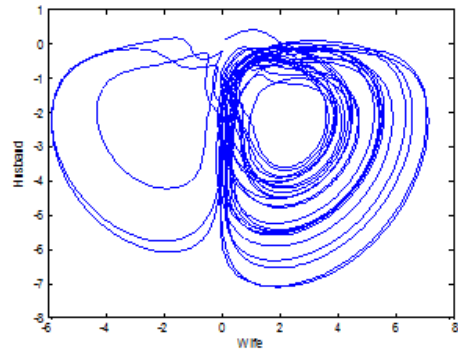

(a)

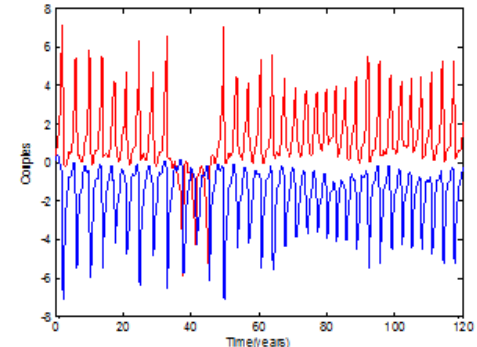

(b)

FigurE 1. (a) The phase portrait and, (b) corresponding time series for those that agreed with the question being asked with $\alpha=0.99$ and $f(t)=0.4 \pi t$.

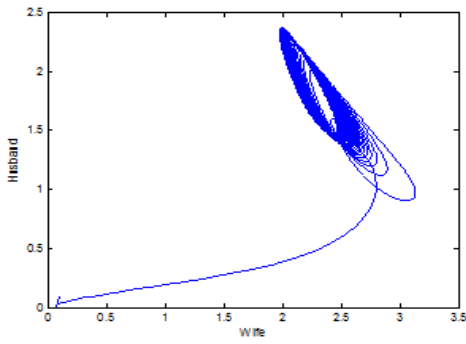

(a)

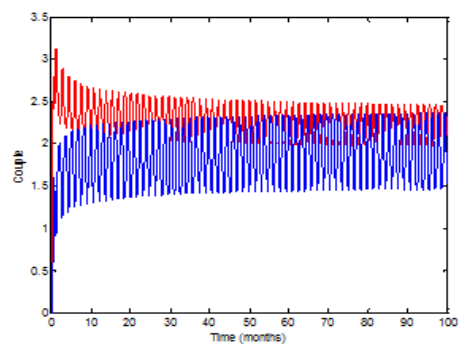

(b)

FIGURE 2. The fractional-order love system showing periodic orbit with $\alpha=0.25$ and $f(t)=0.4 \pi t$ using the same parameters and initial conditions as in Fig. (1). 
Case 2: Investigating the nature of the phase portraits and time series for the couples that disagreed with the questions at order 0.75 and 0.99 , the trajectory of system (Eqn. 1.5) started from the state of indifference and shows the attractor and time response of the couples both in Fig. (3a-b) and (4a-b).

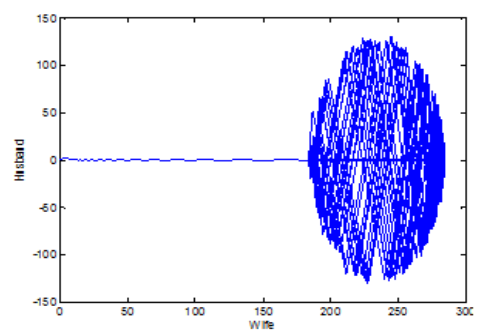

(a)

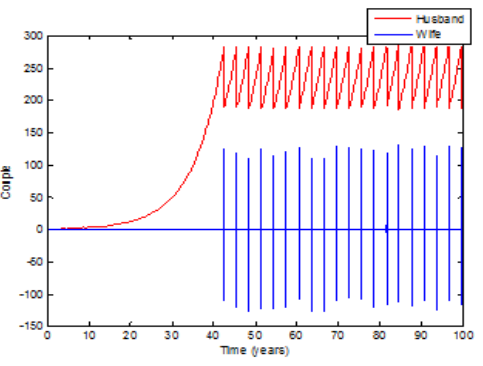

(b)

Figure 3. (a) The phase portrait and, (b) time series for those that disagreed with the question being asked with $\alpha=0.99$ and $f(t)=0.4 \pi t$.

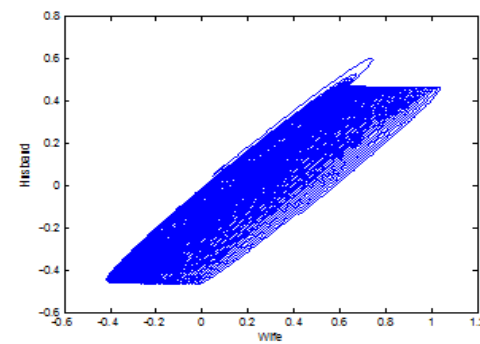

(a)

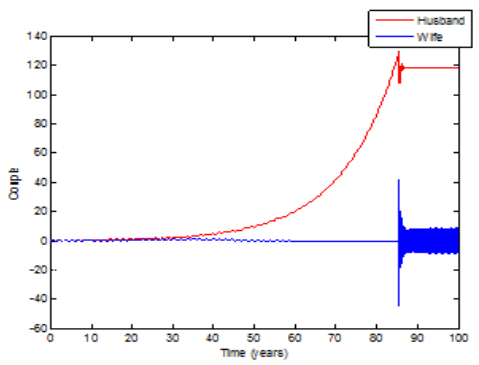

(b)

FIGURE 4. Results of the phase portrait and evolution of the couples that disagreed to the questions with $\alpha=0.75$ and $f(t)=0.4 \pi t$ depicts the time series like a controlled oscillating time series.

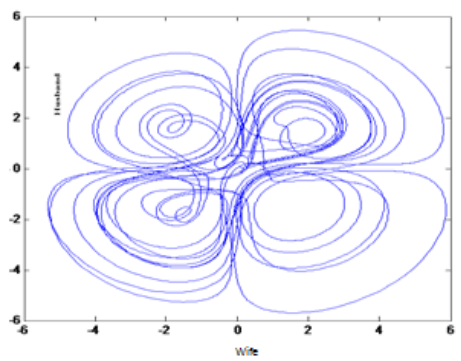

(a)

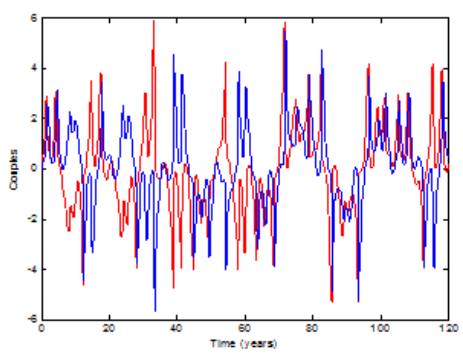

(b)

FigURE 5. Results of the phase portrait (a) and time series plot of couples dynamics with a periodic forcing (b) for those that could not make decision to the questions asked with $\alpha=0.5$ and $f(t)=0.4 \pi t$ at certain parameters and initial conditions. 


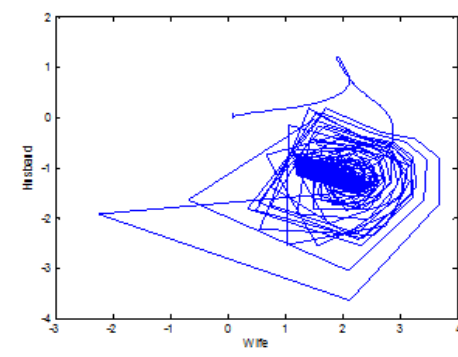

(a)

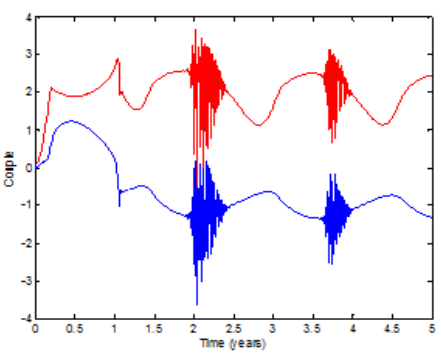

(b)

FIGURE 6. Results of the phase portrait (a) and time series plot of couples dynamics with a periodic forcing (b) for those that could not make decision to the questions asked with $\alpha=0.2$ and $f(t)=0.4 \pi t$.

Case 3: Investigating the nature of the phase portraits and time series for the couples that could not make decision on whether they are happy in their marriage or not. Figure 6 indicates irregular pattern in their marital life at order 0.2 and as the order increases the attractor becomes more chaotic as shown in Figure 5 with order 0.5

\subsubsection{Result 2.}

In analyzing the data, it was discovered that seventeen couples were already divorced out of one hundred samples and due to the fact that fractional differential equation has a special properties called memory effect that is, next state of fractional model depends not only upon its current state but also upon all its historical states therefore changing the order of the equations of a system analyses the chaotic dynamics of the fractional order system. After an initial transient, the solution settles into an irregular oscillation but never repeats exactly.

Case 4: Mathematical analysis was used to study human behaviour in form of equations and the journey of life of seventeen (17) different divorcee out of hundred (100) couples interviewed were captured. But just two couples were cited using the analysis of each couple as parameters in the model with different impact factors of memory. The phase and time series plots are shown.

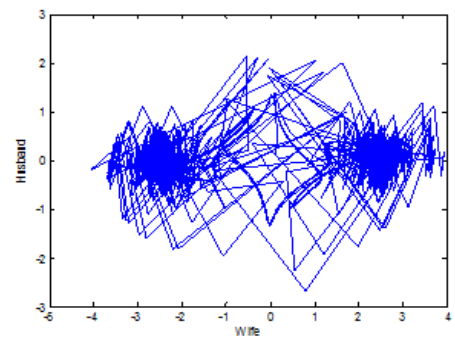

(a)

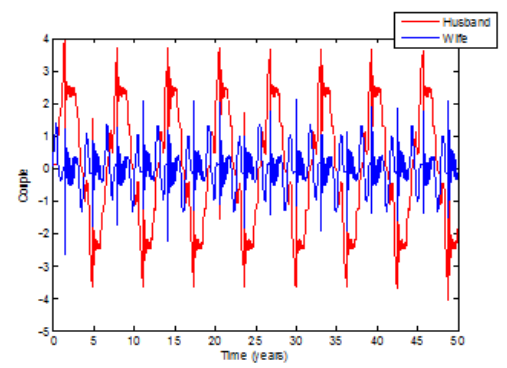

(b)

FIGURE 7. Results of the phase portrait (a) and time evolution of a couple with a periodic forcing, $\alpha=0.5$ and $f(t)=2 \pi t$. The system does not show any plot when $\alpha<0.5$. In this case the parameters are taken from the analysis of couple A. 


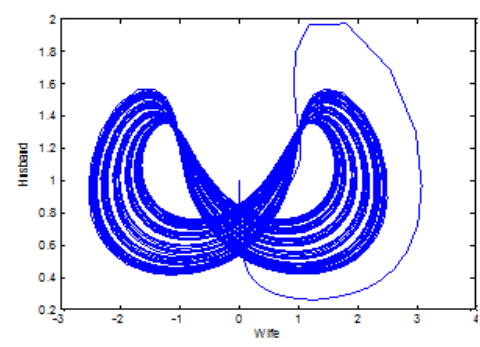

(a)

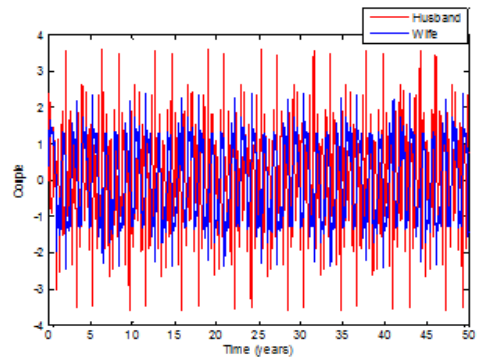

(b)

FigURE 8. Attractor and time series plots of fractional-order love system with $\alpha=0.75$ and $f(t)=2 \pi t$. In this case the parameters are taken from the analysis of couple $A$.

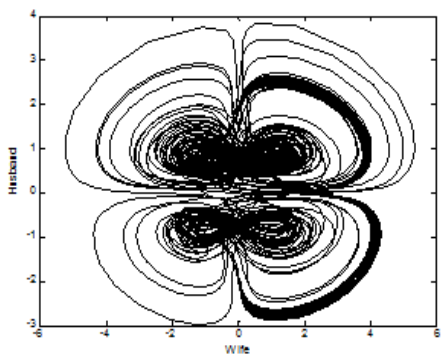

(a)

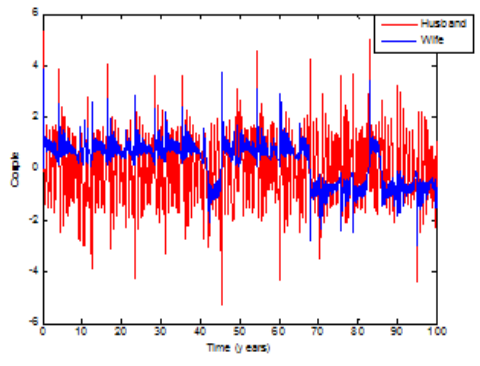

(b)

FiguRE 9. The attractor and time series plots of fractional-order love system of couple $A$, with $\aleph=0.95$ and $f(t)=2 \pi t$ showing the effect of the parameters.

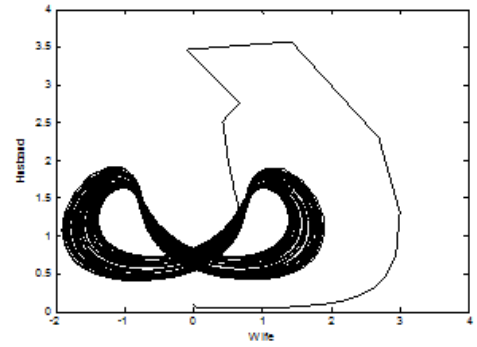

(a)

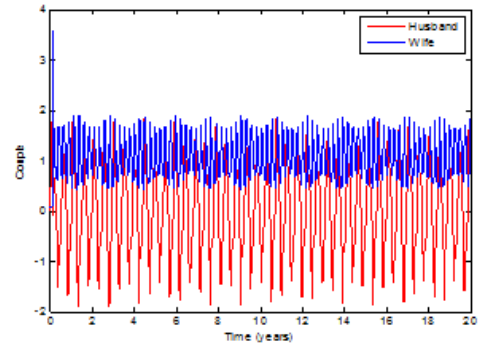

(b)

Figure 10. Attractor and time series plots of fractional-order love system with $\alpha=0.75$ and $f(t)=2 \pi t$. In this case the parameters are taken from the analysis of couple $B$.

\section{Conclusion}

Song and Yang (2009) in their work used Adams Predictor Corrector Scheme because of the specificity of the error estimation bound, efficiency and reliability of the method; they were able to find the existence of chaos in the fractional-order happiness model. Same method was used in this study and the existence of chaos was 


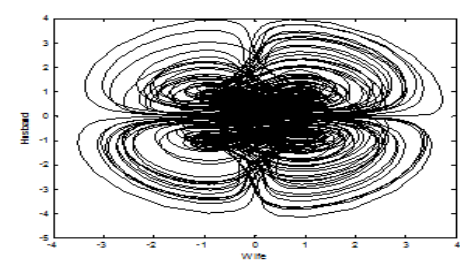

(a)

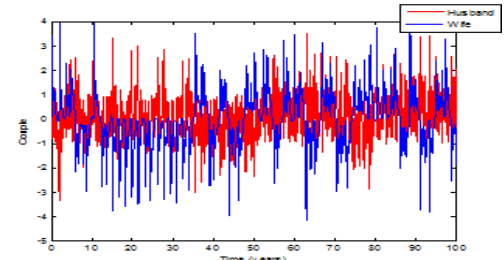

(b)

FiguRE 11. Attractor and time series plots of fractional-order love system with $\alpha=0.95$ and $f(t)=2 \pi t$. In this case the parameters are taken from the analysis of couple $B$.

determined in the modified fractional model of dynamical system for the relationships in marriages which we acquired using the parameters from data analysis with the aid of questionnaire and computer simulation.

It was observed that different couples show diverse behavior with different impact factor of memory.This therefore signifies that the love between couples is affected by many factors. The paper looked into the different phase plots of the couples that agreed, disagreed and could not make decision to the reason for being married. It also probed into the different phase and time series plots of couples that had already divorced.

The phase and time series plots shows that people corresponding to these parameter pairs in the society have complicated and unpredictable feelings in their marital life. Therefore, in order to maintain relationship between couples, they should both give their best to maintain their love status.

\section{References}

[1] F. A. Alawad, E. A. Yousif, and I. A. Arbab, A new technique of Laplace iteration method for solving space-time fractional telegraph equations, International Journal of Differential Equations, Article ID 256593, 10 pages, doi: 10.1155/2013/256593, (2013).

[2] A. Cheriff, and K. Barley, Stochastic nonlinear dynamics of interpersonal and romantic relationships, Applied Mathematics and Computation, 217(13), 6273-6281, doi:10.1016/j.amc.2010.12.117, (2011).

[3] J. M. Gottman, J. D. Murray, C. C. Swanson, R. Tyson, , and K. R. Swanson, The Mathematics of marriage, Cambridge, MA: MIT Press, (2005).

[4] J. Gottman, S. Catherine, and S. Kristin, A general systems theory of marriage: Nonlinear difference equation modeling of marital interaction, Personality and Social Psychology Review 6(4), 326-340, (2002).

[5] A. Gragnani, S. Rinaldi, and F. Gustav, Cyclic dynamics in romantic relationships, International Journal of Bifurcation and Chaos. 7(11), 2611-2619, (1997).

[6] R. Gu, and Y. Xu, Chaos in a fractional-order dynamical model of love and its control In: Li S., Wang X., Okazaki Y., Kawabe J., Murofushi T., Guan L. (Eds) Nonlinear Mathematics for Uncertainty and its Applications, Advances in Intelligent and Soft Computing, vol. 100, pp. 349-356. Springer, Berlin, Heidelberg (2011, January).

[7] W. Liu, and K. Chen, Chaotic behavior in a new fractional-order love triangle system with competition, Journal of Applied Analysis and Computation, 5(1), 103-113.doi:10.11948/2015009, (2015).

[8] F. Mohammadi, Numerical solution of Bagley-Tovik equation using Chebyshev operational matrix of fractional derivative, International Journal of Advances in Applied Mathematics and Mechanics,2(1), 83-91, ISSN:2347-2529, (2014).

[9] O.S Odetunde, and O.A Taiwo, An algorithm for the approximation of fractional differential-algebraic equations with Caputo-type derivatives, Journal of Applied \& Computational Mathematics, 4(5),242, doi: 10.4172/21689679.1000242, (2015).

[10] E. Okyere, F. T. Oduro, S. K. Amponsah, I. K. Dontwi, and N. K. Frempong, Fractional order SIR model with constant population, British Journal of Mathematics and Computer Science, 14(2) 1-12, doi: 10.9734/BJMCS/2016/23017, (2016). 
[11] N. Ozalp, and I. Koca, A fractional order nonlinear dynamical model of interpersonal relationships, Advances in Difference Equations, 2, 1-7, (2012).

[12] R. P. Paul, S. L. Larry, M. G. John, D. N. Michael, and S. Jessica, A mathematical model of psychotherapy: An investigation using dynamic non-linear equations to model the therapeutic relationship, Psychoterapy Research, doi:10.1080/10503307.2011.622314, (2011).

[13] L. Song, and J. Yang, Chaos control and synchronization of dynamical model of happiness with fractional order, Conference proceeding of the 4th IEEE Conference on Industrial Electronics and Applications (ICIEA), Institute of Electrical and Electronics Engineers, Inc. 919924 10.1109/ICIEA.2009.5138330. Retrieved from ieeexplore.ieee.org/document/5138330/,(2009, May 29).

[14] L. Von Bertalanffy, General system theory, New York: Family Psychology, (9), 110-130, (1968).

[15] D. Weigel, and C. Murray, The paradox of stability and change in relationships: What does chaos theory offer for the study of romantic relationships? , 17(3), 425-449, (2000). 